\title{
PROCESSO DE DESENVOLVIMENTO DE PRODUTOS NO VESTUÁRIO: UMA REVISÃO SISTEMÁTICA DE MODELOS DE AUXÍLIO À PRÁTICA PROJETUAL DE PRODUTOS DE MODA
}

\section{CLOTHING PRODUCTS DEVELOPMENT PROCESS: A SYSTEMATIC REVIEW OF MODE TO ASSIST FASHION PRODUCTS DESIGN}

\author{
Thiago Varnier ${ }^{1}$, Diego de Castro Fettermann¹, Giselle Schmidt Alves Díaz Merino²
}

\section{RESUMO:}

As empresas de vestuário vêm intensificando seus esforços em busca da excelência no seu desempenho para atender às necessidades dos usuários, e ao mesmo tempo minimizar seus custos operacionais. Para tanto, é necessário adotar uma forma de trabalho que coordene e favoreça o desenvolvimento de novos produtos no setor. Assim, este artigo tem como objetivo identificar os principais modelos desenvolvidos para auxiliar o processo de desenvolvimento de produtos de moda, por meio de uma revisão sistemática nas bases de dados Scopus, Web of Science, Science Direct, Proquest e Scielo. Foram identificados 973 artigos publicados, que após um processo de filtragem foram submetidos a uma análise bibliométrica e de conteúdo. Os resultados indicam: Estados Unidos, Brasil e China, como países com maiores quantidades de publicações, maioria dos modelos propostos estão destinados às fases de pré-desenvolvimento e desenvolvimento de modo a proporcionar maior adequação das especificidades do usuário ao produto, além de um foco de pesquisa na inserção do usuário ao processo de desenvolvimento. Portanto, com este levantamento foi possível identificar modelos que buscam aprimorar os processos de desenvolvimento de produtos de moda priorizando a pesquisa (identificação dos requisitos de usuário), criação e detalhamento (geração das soluções), desenvolvimento (produção do vestuário), bem como a promoção e comercialização (apresentação da linha de produtos).

PALAVRAS-CHAVE: Design de Moda; Modelos; Desenvolvimento de Produto; Vestuário.

\section{ABSTRACT:}

Clothing companies have been intensifying their efforts to excel in their performance to meet the needs of their users while minimizing their operating costs. Therefore, it is necessary to adopt a form of work that coordinates and favors the development of new products in the sector. Thus, this article aims to identify the main models developed to assist the process of developing fashion products through a systematic review in the Scopus, Web of Science, Science Direct, Proquest and Scielo databases. We identified 973 published articles, which after a filtering process were subjected to a bibliometric and content analysis. The results indicate: United States, Brazil and China, as countries with the largest number of publications, most of the proposed models are destined to the pre-development and development phases in order to provide a better fit of the user specificities to the product, besides a focus. research on user insertion into the development process. Therefore, with this survey it was possible to identify models that seek to improve the processes of development of fashion products prioritizing research (identification of user requirements), creation and detailing (generation of solutions), development (production of clothing), as well as promotion and marketing (product line presentation).

KEYWORDS: Fashion Design; Models; Product Development; Clothing.
Fonte de Financiamento: CAPES

Conflito de Interesse: Declara não haver.

Ética em Pesquisa: Declara não haver necessidade.

Submetido em: 27/06/2020 Aceito em: 25/12/2020

How to cite this article:

VARNIER, T.; FETTERMANN, D.C.; MERINO, G.S.A.D. Processo de desenvolvimento de produtos no vestuário: uma revisão sistemática de modelos de auxílio à prática projetual de produtos de moda. Gestão \& Tecnologia de Projetos. São Carlos, v16, n2, 2021. https://doi.org/10.11606/gtp.v16i2Y.171529 
Processo de desenvolvimento de produtos no vestuário: uma revisão sistemática de modelos de auxílio à prática projetual de produtos de moda

\section{INTRODUÇÃO}

Devido a necessidade de aumentar a competitividade no mercado, as empresas de vestuário vêm intensificando seus esforços em busca da excelência no seu desempenho, atendendo às necessidades dos usuários, e ao mesmo tempo buscando a máxima utilização dos seus recursos, a minimização dos seus custos operacionais e garantindo a qualidade do produto final (MORETTI; BRAGHINI, 2017). Neste sentido, as empresas de vestuário precisam oferecer produtos de sucesso devido à concorrência acirrada (TSE; CHAN, 2016). Para tanto, Fornasieiro e Zangiacomi (2013) afirmam que a adoção de metodologias e tecnologias de produção é de importância decisiva para estas empresas, visto que podem responder proativamente a alta variabilidade das demandas e expectativas dos usuários, bem como melhorar seus processos de desenvolvimento de produtos.

No que tange ao Processo de Desenvolvimento de Produtos (PDP), Silva e Rupansingue (2018, p. 279) definem como "uma série de atividades, desde a geração de ideias até a comercialização do produto, que são interligadas para fornecer novos resultados aos produtos". De forma complementar o PDP ainda pode ser compreendido como uma sequência de tarefas que uma organização emprega para conceber, projetar e comercializar um produto (AYAĞ, 2005). No entanto, na indústria da moda, o desenvolvimento de produtos é um processo crítico e muito desafiador (GUNESOGLU; MERIC, 2007). Isto se deve ao fato de que os produtos da moda são desenvolvidos em linhas sazonais (coleções) em vez de produtos individuais, o que aumenta a necessidade de serem gerenciados simultaneamente, tornando o processo de desenvolvimento ainda mais complexo (MAY-PLUMLEE; LITTLE, 1998). Além disso, este processo passa por várias etapas em um curto espaço de tempo, que vão desde a identificação do problema, criação, materialização até a sua disponibilização no mercado (TREPTOW, 2013).

Back et al. (2008) abordam que para coordenar o desenvolvimento destes produtos em todas estas etapas torna-se importante adotar uma forma de trabalho, que confere vantagens consideráveis em relação ao tempo despendido ao desenvolvimento do produto de vestuário. Atualmente, o setor de fabricação de vestuário está sendo aprimorado e diferentes empresas vem utilizando metodologias para desenvolver novos produtos (SILVA; RUPASINGHE, 2018). No entanto, ainda se encontra restrita a literatura específica sobre a abordagem do Processo de Desenvolvimento de Produtos de vestuário (MORETTI; BRAGHINI, 2017). Sanches (2008), corrobora que o uso de metodologias de design ainda é pouco explorado por gestores empresariais e até mesmo no meio acadêmico e científico da área de moda. A criação de uma metodologia, também entendida como um modelo de referência para o PDP, específico para a indústria do vestuário e adaptada às suas características, é uma forma de estruturar seu PDP, aumentando a performance do processo de desenvolvimento e, consequentemente, o mercado das empresas (MORETTI; BRAGHINI, 2017).

Mediante o panorama apresentado, o estudo do Processo de Desenvolvimento de Produtos de vestuário, ou seja, o fluxo de atividades e as ferramentas, métodos e procedimentos técnicos utilizados nesse processo são fundamentais para garantir qualidade do produto e agilidade no processo. Assim, observou-se uma lacuna de pesquisa quanto a um levantamento dos modelos já desenvolvidos para a área da moda, e quantos deles se destinam ao processo de desenvolvimento, como ferramenta auxiliar ao processo de projeto. Dessa forma, este artigo tem como objetivo realizar um levantamento e identificar os principais modelos desenvolvidos para auxiliar o processo de desenvolvimento de produtos de moda, por meio de uma busca sistemática nas bases de dados Scopus, Web of Science, Science Direct, ProQuest e Scielo. Para tanto, nesta pesquisa, modelos serão enquadrados como qualquer ferramenta, metodologia, método e/ou princípio, apresentado como forma de auxiliar ou guiar o processo de desenvolvimento do produto de moda. 


\section{MATERIAIS E MÉTODOS}

Para atingir o objetivo dessa pesquisa realizou-se uma revisão sistemática utilizando o método proposto por Sampaio e Mancini (2007), os quais descrevem o desenvolvimento da revisão em cinco etapas: (i) Definir a pergunta; (ii) Buscar a evidência; (iii) Revisar e selecionar os estudos; (iv) Analisar a qualidade metodológica dos estudos; e (v) Apresentar os resultados.

Seguindo o método proposto por Sampaio e Mancini (2007), foram definidas as seguintes perguntas de pesquisa: quais são os modelos que auxiliam no processo de desenvolvimento de produtos de vestuário? Em que países foram desenvolvidos? Qual fase de projeto o modelo pretende auxiliar? Quais conceitos e modelos influenciaram o desenvolvimento dos mesmos? E quais são os objetivos de atuação?

A busca pelas evidências se deu em estudos nacionais e internacionais encontrados nas bases de periódicos da Scopus, Web of Science, Science Direct, ProQuest e Scielo. Para tanto, utilizou-

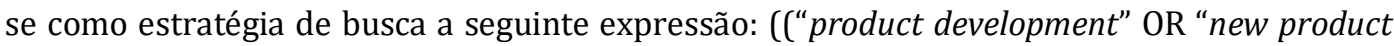
development" OR "development process") AND (fashion OR clothing OR clothes OR garment OR dress) AND (method* OR tools)). A mesma expressão de busca foi utilizada nas cinco bases de dados. Os critérios de inclusão ou exclusão para seleção dos documentos foram:

- Foram incluídos os artigos nos idiomas português, inglês e espanhol, por serem os idiomas dominados pelos autores, para posterior leitura e análise do conteúdo;

- Artigos publicados em periódicos indexados às bases de dados e artigos de anais de congresso que continham título, resumo e/ou palavras-chave que fizessem referência aos termos estudados (métodos e ferramentas para desenvolvimento de produtos do vestuário), sendo excluídos os artigos que não apresentassem relação com o tema proposto, e que não relatassem o funcionamento ou desenvolvimento do modelo de desenvolvimento;

- Os artigos não foram limitados por data de publicação, como também não houve restrição quanto a área de pesquisa registrada na base de dados;

- Foram incluídos os artigos que estavam disponíveis para download no período, com acesso ao texto completo pelos portais da CAPES (Coordenação de Aperfeiçoamento de Ensino Superior), do Google, do Google Scholar ou enviados por e-mail aos autores.

Vale ressaltar que além dos critérios de inclusão e exclusão supracitados, os artigos selecionados passaram por uma leitura dinâmica do documento na integra, sendo excluídos os trabalhos que não apresentassem a descrição do processo de desenvolvimento do modelo, ferramenta ou método desenvolvido. Para organizar e aplicar os critérios de inclusão e exclusão utilizou-se o software EndNote X6®.

Posteriormente para realizar a análise das publicações selecionadas e construir a matriz de síntese, utilizou-se o Microsoft Excel 2016. A síntese dos resultados foi realizada mediante a construção de duas matrizes de análise do conteúdo: (1) referente a análise bibliométrica e a (2) referente a abordagem dos documentos. No que tange a matriz (1) análise bibliométrica compreendeu os tópicos da identificação da publicação: autor, título, ano, país, periódico ou evento científico e o número de citações. Já a matriz (2) de análise do conteúdo seguiu os tópicos propostos por Pichler e Merino (2017):

- Tipo de pesquisa utilizado no artigo: Teórica (descreve o modelo desenvolvido) ou Empírica (relata o modelo em uso, com aplicação prática do modelo); 
- Identificação do modelo: nome, sigla, etapas do modelo, objetivo do modelo, conceitos (conceitos utilizados para seu desenvolvimento) e modelos base (modelos que foram utilizados como base para seu desenvolvimento);

- Tipo de modelo: identifica o tipo de estrutura utilizado para representar o modelo em análise como: Quadro (modelos denominados pelo autor como Framework); Ferramenta (modelos denominados pelo autor como Tool), ou Método (modelos dominados pelo autor como Method ou Methodology);

- Fase de uso: identifica qual momento do desenvolvimento do produto de moda o modelo pode ser utilizado, como: pré desenvolvimento (PRÉ) se utilizado antes da etapa de projeto, como desenvolvimento (DES) se utilizado somente para desenvolver o produto de moda, como pós desenvolvimento (PÓS) se utilizado posteriormente ao desenvolvimento do produto de moda, e como integral (INT) se utilizado antes, durante ou depois do desenvolvimento do produto de moda.

- Tipo de avaliação: identifica o método de projeto utilizado para avaliar o modelo em análise.

\section{RESULTADOS}

Os resultados da revisão sistemática foram separados em dois tópicos: resultados quantitativos que compreendem a análise bibliométrica e resultados qualitativos que compreendem a análise da abordagem dos conteúdos dos documentos selecionados.

\section{RESULTADOS QUANTITATIVOS}

A pesquisa nas bases de dados foi realizada no dia 01 de maio de 2019 e retornou o total de 973 documentos, sendo 457 retirados da base de dados Scopus, 178 da Web of Science, 52 da Science Direct, 286 da ProQuest, e 0 na Scielo. Minerando os documentos duplicados no filtro 1 (F1), 845 restaram para o filtro do título, resumo e palavras-chave (F2). Neste filtro foram descartados 785 artigos que não apresentavam relação com o tema, ou seja, não eram modelos auxiliares ao desenvolvimento do produto de vestuário. Assim, com os 60 artigos restantes, fez-se a busca dos arquivos disponíveis (F3) para realização da leitura dos documentos da integra. Destes, 2 artigos não estavam disponíveis, restando 58 artigos. Após a realização da leitura dinâmica (F4) foram excluídos 45 artigos que não relatavam o desenvolvimento ou funcionamento de um método. Por fim foram selecionados 13 artigos que atenderam aos critérios de seleção especificados nos procedimentos metodológicos. A Figura 1 apresenta 0 processo de filtragem dos documentos.

Figura 1. Fluxograma de seleção de artigos Fonte: Os autores.

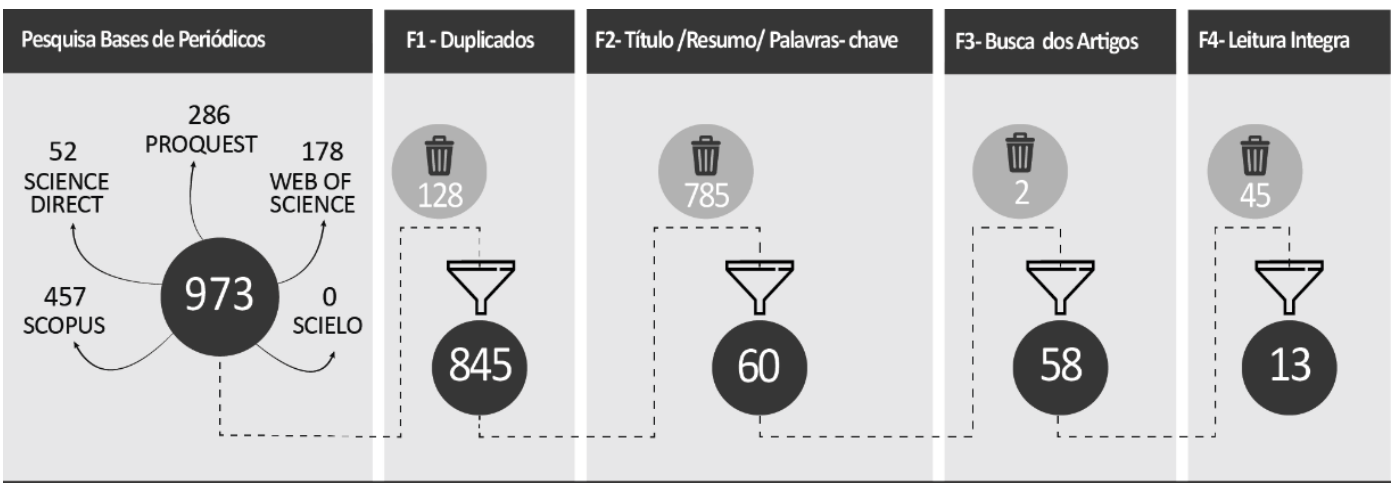


O Quadro 1 apresenta a lista dos 13 artigos selecionados, sendo estes organizados pelo ano (do mais recente para o mais antigo). 0 quadro apresenta o ano, autor, título do trabalho, e citações, as quais foram baseadas no Google Scholar no dia 20 de junho de 2019. 0 artigo "User-oriented product development applied to functional clothing design" (ROSENBLAD-WALLIN, 1985) foi o mais citado, com 88 citações. O segundo mais citado foi "Proactive product development integrating consumer requirements" (MAY-PLUMLEE; LITTLE, 2006), com 75 citações, seguido de "Concurrent engineering for product development in mass customization for the apparel industry" (KINCADE; REGAN; GIBSON, 2007), com 68 citações. 0 quarto mais citado foi "Fuzzy association rule mining for fashion product development" (LEE et al., 2015), com 17 citações, seguido do "A structured approach for customised production in SME collaborative networks" (FORNASIERO; ZANGIACOMI, 2013), com 16 citações. Ainda, 3 artigos apresentam apenas 1 citação, 3 artigos não receberam citações e 1 artigo não foi encontrado o número de citações.

\begin{tabular}{|c|c|c|c|c|}
\hline Ano & Autor & Título & $\begin{array}{l}\text { Periódico ou Evento } \\
\text { Científico }\end{array}$ & Citações \\
\hline 2018 & $\begin{array}{l}\text { Silva; } \\
\text { Rupasinghe }\end{array}$ & $\begin{array}{l}\text { A new apparel product } \\
\text { development framework for } \\
\text { performance clothing industry }\end{array}$ & $\begin{array}{l}\text { International Journal } \\
\text { of Product } \\
\text { Development }\end{array}$ & 1 \\
\hline 2018 & $\begin{array}{l}\text { Morris; } \\
\text { Ashdown, }\end{array}$ & $\begin{array}{l}\text { Partnerships in Practice: } \\
\text { Producing New Design Knowledge } \\
\text { with Users When Developing } \\
\text { Performance Apparel }\end{array}$ & Fashion Practice & 0 \\
\hline 2018 & $\begin{array}{l}\text { Nakayama; } \\
\text { Martins }\end{array}$ & $\begin{array}{l}\text { Fashion Design Methodology } \\
\text { Tools in Products' Development } \\
\text { for People with Disabilities and } \\
\text { Low Mobility }\end{array}$ & $\begin{array}{l}\text { Proceedings of the } \\
\text { 20th Congress of the } \\
\text { International }\end{array}$ & 0 \\
\hline 2017 & $\begin{array}{l}\text { Moretti; } \\
\text { Braghini }\end{array}$ & $\begin{array}{l}\text { Reference model for apparel } \\
\text { product development }\end{array}$ & $\begin{array}{l}\text { Independent Journal } \\
\text { of Management \& } \\
\text { Production }\end{array}$ & 1 \\
\hline 2016 & Tse; Chan & $\begin{array}{l}\text { New approach for fashion design: } \\
\text { Case study of employing user- } \\
\text { oriented method to design } \\
\text { mother-to-be party dress }\end{array}$ & $\begin{array}{l}\text { Research Journal of } \\
\text { Textile and Apparel }\end{array}$ & 0 \\
\hline 2016 & $\begin{array}{l}\text { Mehtälä et } \\
\text { al. }\end{array}$ & $\begin{array}{l}\text { A crowdsourcing model for new } \\
\text { idea development in the fashion } \\
\text { industry }\end{array}$ & $\begin{array}{l}\text { International } \\
\text { Conference on } \\
\text { Service Operations }\end{array}$ & 1 \\
\hline 2015 & Lee et al & $\begin{array}{l}\text { Fuzzy association rule mining for } \\
\text { fashion product development }\end{array}$ & $\begin{array}{l}\text { Industrial } \\
\text { Management \& Data } \\
\text { Systems }\end{array}$ & 17 \\
\hline 2014 & $\begin{array}{l}\text { Maldonado; } \\
\text { Rivera; Caro }\end{array}$ & $\begin{array}{l}\text { Improvement of new product } \\
\text { development process in apparel } \\
\text { SMEs using lean and life cycle }\end{array}$ & $\begin{array}{l}\text { Proceedings of the } \\
\text { XIX Summer School } \\
\text { Francesco Turco }\end{array}$ & NE \\
\hline 2013 & $\begin{array}{l}\text { Fornasiero; } \\
\text { Zangiacomi }\end{array}$ & $\begin{array}{l}\text { A structured approach for } \\
\text { customised production in SME } \\
\text { collaborative networks }\end{array}$ & $\begin{array}{l}\text { International Journal } \\
\text { of Production } \\
\text { Research }\end{array}$ & 16 \\
\hline 2007 & $\begin{array}{l}\text { Kincade; } \\
\text { Regan; } \\
\text { Gibson }\end{array}$ & $\begin{array}{l}\text { Concurrent engineering for } \\
\text { product development in mass } \\
\text { customization for the apparel } \\
\text { industry }\end{array}$ & $\begin{array}{l}\text { International Journal } \\
\text { of Operations \& } \\
\text { Production } \\
\text { Management }\end{array}$ & 68 \\
\hline
\end{tabular}

Quadro 1. Portfólio final dos artigos nacionais e internacionais

Fonte: Os autores.

Legenda:

$\mathrm{NE}=$ não encontrado 


$\begin{array}{|lllll|}2006 & \begin{array}{l}\text { Johnson- } \\ \text { Leslie; } \\ \text { Gaskill }\end{array} & \begin{array}{l}\text { Small business apparel retailing in } \\ \text { Jamaica: an exploratory } \\ \text { investigation into product } \\ \text { development processes and } \\ \text { practices }\end{array} & \begin{array}{l}\text { The Qualitative } \\ \text { Report }\end{array} & 5 \\ 2006 & \text { May- } & \begin{array}{l}\text { Proactive product development } \\ \text { integrating consumer } \\ \text { requirements }\end{array} & \begin{array}{l}\text { International Journal } \\ \text { of Clothing Science } \\ \text { and Technology }\end{array} & 75 \\ & \begin{array}{l}\text { Plumlee; } \\ \text { Little }\end{array} & \begin{array}{l}\text { Applied ergonomics } \\ \text { Rosenblad- } \\ \text { Wallin }\end{array} & \begin{array}{l}\text { User-oriented product } \\ \text { development applied to functional } \\ \text { clothing design }\end{array} & 88 \\ \end{array}$

Com relação a distribuição dos artigos nos anos de publicação, percebe-se um aparente aumento do número e da frequência nos últimos anos. Até 2007 houve a ocorrência de apenas quatro artigos (ROSENBLAD-WALLIN, 1985); (MAY-PLUMLEE; LITTLE, 2006); (JOHNSONLESLIE; GASKILL, 2006); (KINCADE; REGAN; GIBSON, 2007) abordando modelos a fim de guiar o desenvolvimento de produtos de moda, cujo período compreende as três publicações mais citadas, conforme observado no Quadro 1. Posteriormente, de 2007 a 2013, não foi identificado nenhum modelo. A partir de 2013, o assunto apresenta crescimento no que tange a regularidade de publicações, com ocorrência anual, bem como de dois a três artigos por ano.

O país com maior número de publicação é os Estados Unidos (4 artigos), seguido do Brasil (2) e China (2). Salienta-se que nos países como Siri Lanka, Suécia, Finlândia, Colômbia e Itália, foi encontrado apenas uma publicação. Os dados completos são apresentados na Tabela 1. Cabe salientar que somente o país do primeiro autor foi considerado nesta análise.

Tabela 1. Distribuição do número de publicações por ano e país do primeiro autor

Fonte: Os autores.

\begin{tabular}{|c|c|c|c|c|c|c|c|c|c|}
\hline Ano & $\frac{\overline{\bar{n}}}{\bar{\Phi}}$ & : & $\begin{array}{l}\frac{\pi}{0} \\
\frac{0}{0} \\
\frac{\text { O0 }}{0}\end{array}$ & 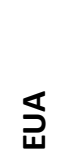 & 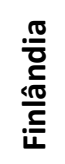 & $\frac{\mathbb{\pi}}{\frac{\pi}{\pi}}$ & $\begin{array}{l}\frac{\pi}{c} \\
\frac{\pi}{J} \\
\frac{D}{n}\end{array}$ & $\frac{\pi}{\frac{\pi}{d}}$ & Total \\
\hline 1985 & & & & & & & & 1 & 1 \\
\hline 2006 & & & & 2 & & & & & 2 \\
\hline 2007 & & & & 1 & & & & & 1 \\
\hline 2013 & & & & & & 1 & & & 1 \\
\hline 2014 & & & 1 & & & & & & 1 \\
\hline 2015 & & 1 & & & & & & & 1 \\
\hline 2016 & & 1 & & & 1 & & & & 2 \\
\hline 2017 & 1 & & & & & & & & 1 \\
\hline 2018 & 1 & & & 1 & & & 1 & & 3 \\
\hline Total & 2 & 2 & 1 & 4 & 1 & 1 & 1 & 1 & 13 \\
\hline
\end{tabular}

Por fim, quanto aos periódicos analisados percebe-se que não há um periódico específico que contemple essa temática reunida, sendo que nenhum periódico se repetiu na análise deste estudo. 


\section{RESULTADOS QUALITATIVOS}

No Quadro 2 são apresentados os 13 modelos selecionados, bem como o levantamento quanto ao tipo de pesquisa, de modelo e de métodos de avaliação.

\begin{tabular}{|c|c|c|c|c|c|c|c|}
\hline Autor & Ano & Nome do Modelo & Sigla & $\begin{array}{l}\text { Tipo de } \\
\text { Pesquisa }\end{array}$ & $\begin{array}{l}\text { Tipo de } \\
\text { Modelo }\end{array}$ & $\begin{array}{l}\text { Tipo de } \\
\text { Avaliação }\end{array}$ & $\begin{array}{l}\text { Quadro 2. Modelos, tipo } \\
\text { de pesquisa, tipo de } \\
\text { modelo e tipo de }\end{array}$ \\
\hline $\begin{array}{l}\text { Silva; } \\
\text { Rupasinghe }\end{array}$ & 2018 & $\begin{array}{l}\text { Estrutura de } \\
\text { desenvolvimento } \\
\text { de produtos para } \\
\text { roupas de } \\
\text { desempenho }\end{array}$ & ---- & Teórica & Quadro & Não relata & $\begin{array}{l}\text { avaliação adotados na } \\
\text { pesquisa } \\
\text { Fonte: Os autores. }\end{array}$ \\
\hline $\begin{array}{l}\text { Morris; } \\
\text { Ashdown, }\end{array}$ & 2018 & $\begin{array}{l}\text { Criação de } \\
\text { conhecimento de } \\
\text { projeto no } \\
\text { processo de } \\
\text { design de } \\
\text { vestuário de } \\
\text { desempenho }\end{array}$ & ---- & Teórica & Quadro & Não relata & \\
\hline $\begin{array}{l}\text { Nakayama; } \\
\text { Martins }\end{array}$ & 2018 & $\begin{array}{l}\text { Ferramenta } \\
\text { metodológica } \\
\text { para contribuir no } \\
\text { processo de } \\
\text { design de } \\
\text { produtos de moda } \\
\text { para pessoas com } \\
\text { algum tipo de } \\
\text { mobilidade } \\
\text { reduzida }\end{array}$ & ---- & Teórica & Ferramenta & Não relata & \\
\hline $\begin{array}{l}\text { Moretti; } \\
\text { Braghini }\end{array}$ & 2017 & $\begin{array}{l}\text { Modelo de } \\
\text { referência para o } \\
\text { processo de } \\
\text { desenvolvimento } \\
\text { de produtos de } \\
\text { vestuário }\end{array}$ & APDP & Teórica & Método & Não relata & \\
\hline Tse; Chan & 2016 & $\begin{array}{l}\text { Método orientado } \\
\text { pelo usuário }\end{array}$ & ---- & Empírica & Método & $\begin{array}{l}\text { Estudo de } \\
\text { caso }\end{array}$ & \\
\hline $\begin{array}{l}\text { Mehtälä et } \\
\text { al. }\end{array}$ & 2016 & $\begin{array}{l}\text { Modelo de } \\
\text { crowdsourcing } \\
\text { proposto para o } \\
\text { desenvolvimento } \\
\text { de novas ideias na } \\
\text { indústria da moda }\end{array}$ & ---- & Teórica & Método & Não relata & \\
\hline Lee et al & 2015 & $\begin{array}{l}\text { Mineração de } \\
\text { regras de } \\
\text { associação difusa }\end{array}$ & FARM & Empírica & Quadro & $\begin{array}{l}\text { Estudo de } \\
\text { caso }\end{array}$ & \\
\hline $\begin{array}{l}\text { Maldonado; } \\
\text { Rivera; Caro }\end{array}$ & 2014 & $\begin{array}{l}\text { Metodologia de } \\
\text { Implementação } \\
\text { LPD para setor do } \\
\text { vestuário }\end{array}$ & LPD & Empírica & Método & $\begin{array}{l}\text { Estudo de } \\
\text { caso }\end{array}$ & \\
\hline
\end{tabular}




\begin{tabular}{|c|c|c|c|c|c|c|}
\hline $\begin{array}{l}\text { Fornasiero; } \\
\text { Zangiacomi }\end{array}$ & 2013 & $\begin{array}{l}\text { Modelo de } \\
\text { referência } \\
\text { CoReNet }\end{array}$ & CoRM & Teórica & Quadro & $\begin{array}{l}\text { Reuniões e } \\
\text { Workshops } \\
\text { específicos }\end{array}$ \\
\hline $\begin{array}{l}\text { Kincade; } \\
\text { Regan; } \\
\text { Gibson }\end{array}$ & 2007 & $\begin{array}{l}\text { Processo de } \\
\text { desenvolvimento } \\
\text { de produtos } \\
\text { focado no } \\
\text { consumidor }\end{array}$ & ---- & Empírica & Método & $\begin{array}{l}\text { Estudo de } \\
\text { caso }\end{array}$ \\
\hline $\begin{array}{l}\text { Johnson- } \\
\text { Leslie; } \\
\text { Gaskill }\end{array}$ & 2006 & $\begin{array}{l}\text { Modelo de } \\
\text { Desenvolvimento } \\
\text { de Produtos }\end{array}$ & ---- & Empírica & Método & $\begin{array}{l}\text { Estudo de } \\
\text { caso }\end{array}$ \\
\hline $\begin{array}{l}\text { May- } \\
\text { Plumlee; } \\
\text { Little }\end{array}$ & 2006 & $\begin{array}{l}\text { Modelo de } \\
\text { desenvolvimento } \\
\text { proativo de } \\
\text { produtos que } \\
\text { integra os } \\
\text { requisitos do } \\
\text { consumidor }\end{array}$ & PPDICR & Teórica & Ferramenta & Não relata \\
\hline $\begin{array}{l}\text { Rosenblad- } \\
\text { Wallin }\end{array}$ & 1985 & $\begin{array}{l}\text { Desenvolvimento } \\
\text { de produto } \\
\text { orientado ao } \\
\text { usuário }\end{array}$ & ---- & Empírica & Método & $\begin{array}{l}\text { Estudo de } \\
\text { caso }\end{array}$ \\
\hline
\end{tabular}

Quanto ao tipo de pesquisa, observou-se que o número de pesquisas empíricas é praticamente igual ao número de pesquisa teóricas, sendo seis (6) e sete (7) respectivamente. Neste sentido as pesquisas empíricas tratam, principalmente, da apresentação de estudos de caso do modelo em uso. Já as pesquisas teóricas tratam da descrição do modelo, seu funcionamento e embasamento na literatura, com apresentação de outros modelos semelhantes ou que serviram de base para a sua construção.

Em relação ao tipo de modelo, sete (7) são abordados pelos autores como método ou metodologia (MORETTI; BRAGHINI, 2017; TSE; CHAN, 2016; MEHTÄLÄ et al., 2016; MALDONADO; RIVERA; CARO, 2014; KINCADE; REGAN; GIBSON, 2007; JOHNSON-LESLIE; GASKILL, 2006; ROSENBLAD-WALLIN, 1985), quatro (4) foram identificados como Quadros "Framework" no inglês (SILVA; RUPASINGHE, 2018; MORRIS; ASHDOWN, 2018; LEE et al.,2015; FORNASIERO; ZANGIACOMI, 2013 ), e dois (2) como ferramenta (NAKAYAMA; MARTINS, 2018; MAY-PLUMLEE; LITTLE, 2006). Quanto ao tipo de avaliação do modelo proposto, observou-se a preferência por estudos de caso, representando seis (6) artigos. Apenas um modelo (FORNASIERO; ZANGIACOMI, 2013) apresenta como forma de avaliação, técnicas de reuniões e workshops específicos. Em 6 artigos não foi relatado a forma de avaliação dos modelos e cabe ressaltar que todos apresentam a pesquisa teórica como tipo de pesquisa.

No Quadro 3 são apresentados os 13 modelos analisados, com identificação das etapas, princípios ou dimensões que compreendem o modelo, as fases de uso no PDP de produto de moda, os conceitos e, por fim, os modelos já existentes que embasaram seu desenvolvimento. 


\begin{tabular}{|c|c|c|c|c|}
\hline Modelo & Etapas & $\begin{array}{l}\text { Fase } \\
\text { de } \\
\text { uso }\end{array}$ & Conceitos & Modelos base \\
\hline $\begin{array}{l}\text { Criação de } \\
\text { conhecimento de } \\
\text { projeto no } \\
\text { processo de } \\
\text { design de } \\
\text { vestuário de } \\
\text { desempenho }\end{array}$ & $\begin{array}{l}\text { Princípios: } \\
\text { Usuários } \\
\text { 1. Socialização- tácito á } \\
\text { tácito; } \\
\text { 2. Externalização - tácito } \\
\text { para explicito; } \\
\text { Membros equipes: } \\
\text { 3.Internalização- explicito } \\
\text { ao tácito; } \\
\text { 4. Combinação - explicito } \\
\text { para explicito. }\end{array}$ & $\begin{array}{l}\text { PRÉ } \\
\text { DES }\end{array}$ & $\begin{array}{l}\text { Conhecimento } \\
\text { tácito e explícito. }\end{array}$ & $\begin{array}{l}\text { Teoria de } \\
\text { Criação de } \\
\text { Conhecimento } \\
\text { de Nonaka } \\
\text { (1994) }\end{array}$ \\
\hline $\begin{array}{l}\text { Ferramenta } \\
\text { metodológica } \\
\text { para contribuir no } \\
\text { processo de } \\
\text { design de } \\
\text { produtos de } \\
\text { moda para } \\
\text { pessoas com } \\
\text { algum tipo de } \\
\text { mobilidade } \\
\text { reduzida }\end{array}$ & $\begin{array}{l}\text { 1. Requisitos gerais de } \\
\text { vestuário e sua } \\
\text { especificação; } \\
\text { 2. Requisitos conceituais; } \\
\text { 3. Requisitos práticos; } \\
\text { 4. Requisitos relacionados } \\
\text { a medições e modelagem; } \\
\text { 5. Requisitos de costura; } \\
\text { 6. Requisitos de materiais. }\end{array}$ & DES & $\begin{array}{l}\text { Design centrado } \\
\text { no usuário; } \\
\text { Ergonomia; } \\
\text { Design Universal, } \\
\text { Usabilidade. }\end{array}$ & $\begin{array}{l}\text { Bonsiepe } \\
\text { (1984); Lobach } \\
\text { (2008); Munari } \\
\text { (2008), Back et } \\
\text { al. (2008); } \\
\text { Montemezzo } \\
\text { (2003); } \\
\text { lida (2005); } \\
\text { Martins } \\
\text { (2005). }\end{array}$ \\
\hline $\begin{array}{l}\text { Modelo de } \\
\text { referência para o } \\
\text { processo de } \\
\text { desenvolvimento } \\
\text { de produtos de } \\
\text { vestuário }\end{array}$ & $\begin{array}{l}\text { 1. Pré-desenvolvimento } \\
\text { (planejamento de coleção } \\
\text { e planejamento do } \\
\text { portfólio de produtos); } \\
\text { 2. Desenvolvimento } \\
\text { (pesquisa de tendência de } \\
\text { mercado; definição do } \\
\text { conceito; detalhamento; } \\
\text { pré-produção; } \\
\text { lançamento da coleção); } \\
\text { 3. Pós-desenvolvimento } \\
\text { (acompanhar } \\
\text { produto/processo). }\end{array}$ & INT & ---- & $\begin{array}{l}\text { Rozenfeld } \\
\text { (2006) (base } \\
\text { para a } \\
\text { estrutura) ; } \\
\text { Gaskill (1992), } \\
\text { Lamb e Kallal } \\
\text { (1992), May- } \\
\text { Plumlee e } \\
\text { Little (1998), } \\
\text { Wickett et al. } \\
\text { (1999), Rench } \\
\text { (2002), } \\
\text { Montemezzo } \\
\text { (2003) e } \\
\text { Pitimanneyaku } \\
\text { I et al. (2004) }\end{array}$ \\
\hline $\begin{array}{l}\text { Método } \\
\text { orientado pelo } \\
\text { usuário }\end{array}$ & $\begin{array}{l}\text { 1. Grupo focal com } \\
\text { clientes alvos para coletar } \\
\text { as informações; } \\
\text { 2. Decompor as } \\
\text { informações em } \\
\text { declarações únicas; } \\
\text { 3. Interpretação das } \\
\text { declarações (por meio da } \\
\text { diagramação por } \\
\text { afinidade); 4. requisitos } \\
\text { de projetos }\end{array}$ & PRÉ & $\begin{array}{l}\text { Design Centrado } \\
\text { no Usuário. }\end{array}$ & ---- \\
\hline
\end{tabular}

Quadro 3. Análise dos modelos quanto as etapas, fases de uso, conceitos e modelos base

Fonte: Os autores. 


\begin{tabular}{|c|c|c|c|c|}
\hline $\begin{array}{l}\text { Modelo de } \\
\text { crowdsourcing } \\
\text { proposto para o } \\
\text { desenvolvimento } \\
\text { de novas ideias } \\
\text { na indústria da } \\
\text { moda }\end{array}$ & $\begin{array}{l}\text { 1. Plataforma (marca } \\
\text { independente) recebe as } \\
\text { ideias, marcações, } \\
\text { votações coletivas, } \\
\text { alternações; } \\
\text { 2. Filtro das informações; } \\
\text { 3. Avaliação por } \\
\text { especialistas; } \\
\text { 4. Seleção das } \\
\text { alternativas; } \\
\text { 5. Produção dos novos } \\
\text { produtos. }\end{array}$ & $\begin{array}{l}\text { PRÉ } \\
\text { DES }\end{array}$ & $\begin{array}{l}\text { Crowdsourcing } \\
\text { (ideias e votação } \\
\text { coletiva) para } \\
\text { valorizar o cliente } \\
\text { e suas opiniões. }\end{array}$ & ---- \\
\hline $\begin{array}{l}\text { Mineração de } \\
\text { regras de } \\
\text { associação difusa }\end{array}$ & $\begin{array}{l}\text { 1. Identificação de } \\
\text { parâmetros; } \\
\text { 2. Definições das } \\
\text { características fuzzy dos } \\
\text { parâmetros; } \\
\text { 3. Gerações de regras de } \\
\text { associação; } \\
\text { 4. Avaliação de regra. }\end{array}$ & PRÉ & $\begin{array}{l}\text { Voz do cliente; } \\
\text { Mineração de } \\
\text { dados. }\end{array}$ & ---- \\
\hline $\begin{array}{l}\text { Metodologia de } \\
\text { Implementação } \\
\text { LPD para setor do } \\
\text { vestuário }\end{array}$ & $\begin{array}{l}\text { 1. Definição da forma de } \\
\text { aplicar o novo PDS na } \\
\text { organização; } \\
\text { 2. Início do Projeto; } \\
\text { 3. Avaliação detalhada e } \\
\text { melhorias preliminares; } \\
\text { 4. Projeto detalhado do } \\
\text { PDS; } \\
\text { 5. Testando o novo } \\
\text { sistema; } \\
\text { 6. Melhorar o design do } \\
\text { sistema; } \\
\text { 7. Validação da } \\
\text { metodologia; } \\
\text { 8. Implantação externa e } \\
\text { flexibilização; } \\
\text { 9. Desenvolvimento de } \\
\text { coleções. }\end{array}$ & PRÉ & $\begin{array}{l}\text { Sistemas } \\
\text { integrados de } \\
\text { gestão; } \\
\text { Design Ecológico; } \\
\text { Macroergonomia. }\end{array}$ & $\begin{array}{l}\text { ISO 31000; } \\
\text { Pardy; Andrew } \\
\text { (2010); } \\
\text { Tool Kit } \\
\text { (EPA,2007); } \\
\text { Methodologic } \\
\text { al Guide for } \\
\text { Reconversion } \\
\text { Plans to Clean } \\
\text { Technologies } \\
\text { (MINAMBIENT } \\
\text { E, 2011). }\end{array}$ \\
\hline $\begin{array}{l}\text { Modelo de } \\
\text { referência } \\
\text { CoReNet }\end{array}$ & $\begin{array}{l}\text { 1. Suporte para análise de } \\
\text { mercado; } \\
\text { 2. Definição de coleção; } \\
\text { 3. Design do produto com } \\
\text { modelagem CAD; } \\
\text { 4. Planejamento de } \\
\text { processo, apoio e suporte; } \\
\text { 5. Tradicional suporte de } \\
\text { compras para grupos } \\
\text { específicos de clientes; } \\
\text { 6. Visualização online, } \\
\text { configuração e aquisição } \\
\text { do produto; } \\
\text { 7. Processamento do } \\
\text { pedido do cliente; }\end{array}$ & INT & $\begin{array}{l}\text { Design Centrado } \\
\text { no Usuário. }\end{array}$ & $\begin{array}{l}\text { SMART (FILOS; } \\
\text { BANAHAN, } \\
\text { 2001) }\end{array}$ \\
\hline
\end{tabular}




\begin{tabular}{|c|c|c|c|c|}
\hline & $\begin{array}{l}\text { 8. Planejamento } \\
\text { colaborativo de processo; } \\
\text { 9. Planejamento } \\
\text { colaborativo de produção } \\
\text { ao controle; } \\
\text { 10. Monitoramento de } \\
\text { parceiros e rastreamento } \\
\text { de suporte; } \\
\text { 11. Produção de } \\
\text { vestuário; } \\
\text { 12. Processo de produção } \\
\text { de calçados } \\
\text { personalizados. }\end{array}$ & & & \\
\hline $\begin{array}{l}\text { Processo de } \\
\text { desenvolvimento } \\
\text { de produtos } \\
\text { focado no } \\
\text { consumidor }\end{array}$ & $\begin{array}{l}\text { 1. Custo; } \\
\text { 2. Tecnologias de } \\
\text { habilitação; } \\
\text { 3. Uso do Ciclo de Vida - } \\
\text { inspeção; } \\
\text { 4. Uso do ciclo de vida - } \\
\text { manutenção; } \\
\text { 5. Uso do ciclo de vida - } \\
\text { confiabilidade; } \\
\text { 6. Fabricação; } \\
\text { 7. Qualidade. }\end{array}$ & INT & $\begin{array}{l}\text { Engenharia } \\
\text { Simultânea; } \\
\text { Design Centrado } \\
\text { no Usuário. }\end{array}$ & $\begin{array}{l}\text { Balasubramani } \\
\text { an, (2001) } \\
\text { Crow (1992) } \\
\text { Parsaei; } \\
\text { Sullivan, } \\
\text { (1993) } \\
\text { Turino (1992) }\end{array}$ \\
\hline $\begin{array}{l}\text { Modelo de } \\
\text { Desenvolvimento } \\
\text { de Produtos }\end{array}$ & $\begin{array}{l}\text { 1. Pré } \\
\text { 1.1 Ideias baseadas em } \\
\text { pesquisa; } \\
\text { 1.2. Esboçar a ideia de } \\
\text { design; } \\
\text { 1.3. Escolha e } \\
\text { fornecimento de tecidos; } \\
\text { 1.4. Refinar e finalizar as } \\
\text { ideias; } \\
\text { 2. Adopção } \\
\text { 2.1. Elaborar padrão para } \\
\text { a ideia de linha; } \\
\text { 2.2 Primeiro cálculo } \\
\text { estimado da ideia de } \\
\text { linha; } \\
\text { 2.3 Criação e avaliação de } \\
\text { amostras; } \\
\text { 3. Pós } \\
\text { 3.1 Refine o padrão } \\
\text { conforme surgem as } \\
\text { necessidades; } \\
\text { 3.2 Produzir e apresentar } \\
\text { linha (s) de produto. }\end{array}$ & INT & ---- & $\begin{array}{l}\text { Wickett et al. } \\
\text { (1999) }\end{array}$ \\
\hline $\begin{array}{l}\text { Modelo de } \\
\text { desenvolvimento } \\
\text { proativo de } \\
\text { produtos que } \\
\text { integra os }\end{array}$ & $\begin{array}{l}\text { 1. Pesquisas / Painéis } \\
\text { comerciais; } \\
\text { 2. Previsões de tendências } \\
\text { comerciais; } \\
\text { 3. Pesquisa Etnográfica; } \\
\text { 4. Modelos de ajuste; }\end{array}$ & DES & $\begin{array}{l}\text { Design Centrado } \\
\text { do Usuário. }\end{array}$ & $\begin{array}{l}\text { EBM Model } \\
\text { (ENGLE et al., } \\
\text { 1995); } \\
\text { NICPPD (MAY- } \\
\text { PLUMLEE; } \\
\text { LITTLE, 1998). }\end{array}$ \\
\hline
\end{tabular}




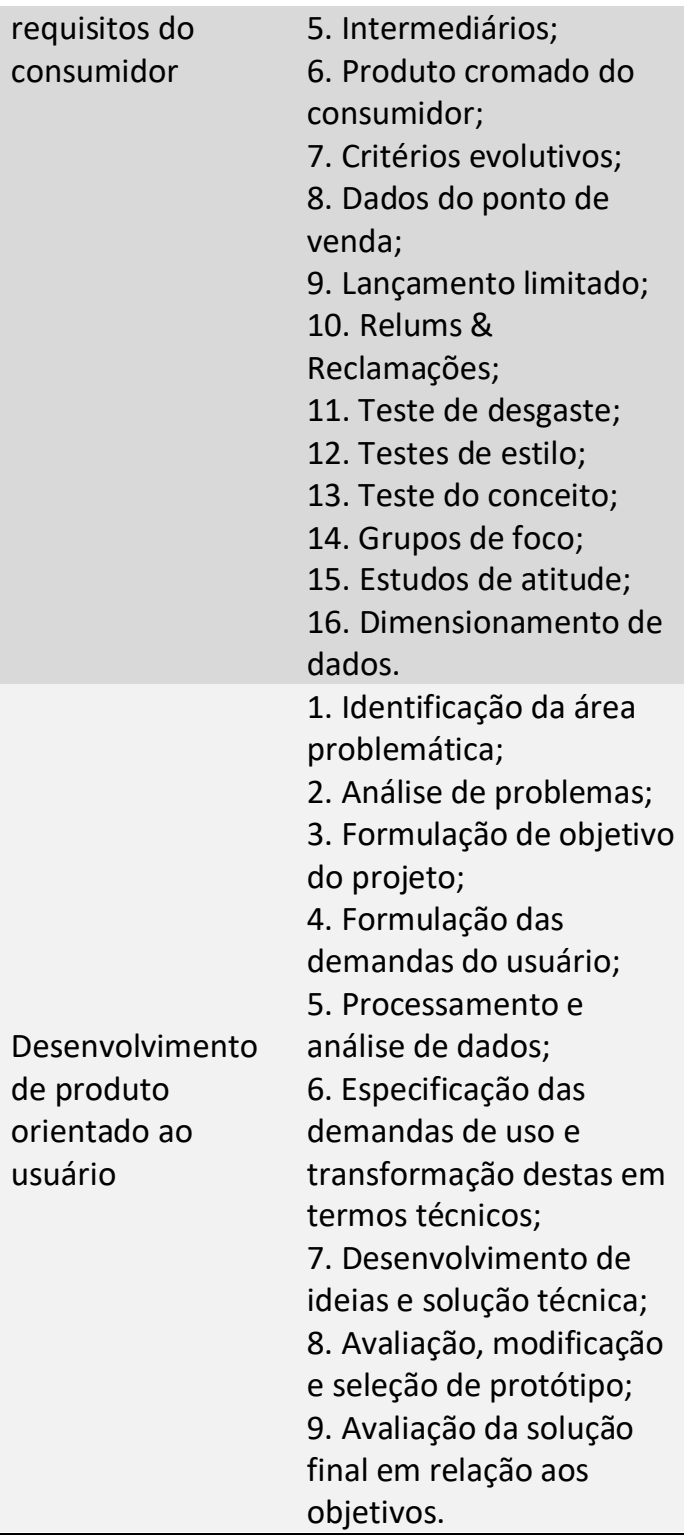

Como pode ser observado no Quadro 3, os modelos são estruturados com no mínimo 3 e no máximo 16 etapas, com predominância para 4, 3, 7 e 9 etapas. Um fator importante a ser destacado é a preocupação da incorporação do usuário no processo de desenvolvimento do produto, identificado em nove (9) dos modelos analisados (SILVA; RUPASINGHE, 2018; NAKAYAMA; MARTINS, 2018; TSE; CHAN, 2016; MEHTÄLÄ et al.,2016; LEE et al.,2015; FORNASIERO; ZANGIACOMI, 2013; KINCADE; REGAN; GIBSON, 2007; MAY-PLUMLEE; LITTLE, 2006; ROSENBLAD-WALLIN, 1985). Ainda cabe ressaltar que alguns modelos enfatizam a observação e análise da necessidade do usuário, além da análise de mercado (SILVA; RUPASINGHE, 2018; TSE; CHAN, 2016; MEHTÄLÄ et al.,2016; LEE et al.,2015; MAY-PLUMLEE; LITTLE, 2006; ROSENBLAD-WALLIN, 1985).

Os conceitos utilizados como referência para o desenvolvimento dos modelos, influenciam diretamente nas abordagens observadas nas etapas supracitadas. Como apresentado no Quadro 3, as principais referências advêm do Design Centrado no Usuário, Design Universal, Usabilidade, Ergonomia, Macroergonomia, Design Ecológico, Crowdsourcing, Engenharia do 
Produto e Engenharia Simultânea. Neste sentido percebe-se uma centralização de estratégias de desenvolvimento dos modelos centrada nas necessidades do usuário.

No que tange as fases de uso, quatro (4) modelos são aplicados no processo inteiro, contemplando as fases de pré-desenvolvimento, desenvolvimento e pós-desenvolvimento, quatro (4) modelos na fase de pré-desenvolvimento, três (3) modelos nas fases de prédesenvolvimento e desenvolvimento e dois (2) modelos na fase de desenvolvimento. Na fase de pré-desenvolvimento se concentram um número maior de modelos do tipo Quadro (3 casos), na fase de desenvolvimento se concentram um número maior de modelos do tipo Ferramentas ( 2 casos) e no processo inteiro se concentra um número maior de modelos do tipo método (4 casos).

Em relação aos modelos utilizados como base, nem todos os modelos analisados apresentaram as referências utilizadas. Alguns modelos chaves foram citados, como precursores de outros modelos, são eles: NICPPD (MAY-PLUMLEE; LITTLE, 1998), que influenciou o desenvolvimento do modelo PPDICR (MAY-PLUMLEE; LITTLE, 2006) como também do modelo APDP (MORETTI; BRAGHIN, 2017). 0 modelo de (WICKETT et al., 1999) influenciou o a proposta do Modelo de Desenvolvimento de Produto (JOHNSON-LESLIE; GASKILL, 2006), bem como o modelo APDP (MORETTI; BRAGHIN, 2017). 0 modelo proposto por Montemezzo (2003) também influenciou o modelo Ferramenta metodológica para contribuir no processo de design de produtos de moda para pessoas com algum tipo de mobilidade reduzida (NAKAYAMA; MARTINS, 2018) e o modelo APDP (MORETTI; BRAGHIN, 2017). Assim, pode-se deduzir que estes modelos base, se encontram mais consolidados neste âmbito. Ainda, na análise dos modelos citados como base, também se observa referências aos modelos propostos por Back et al. (2008) e Rozenfeld et al. (2006) nos conceitos e abordagens de desenvolvimento de produtos.

No Quadro 4 são apresentados os objetivos de cada modelo de desenvolvimento analisado. Observou-se que há um predomínio nas propostas dos modelos em adequar as especificidades do usuário, ou seja, integrar as necessidades do consumidor aos requisitos de projetos (SILVA; RUPASINGHE, 2018), aprimorar e otimizar o processo de design (NAKAYAMA; MARTINS, 2018), gerar ideias diferenciadas para atender melhor aos consumidores (MEHTÄLÄ et al.,2016), bem como utilizar as informações do consumidor para o desenvolvimento do produto (MAY-PLUMLEE; LITTLE, 2006), além de atender as expectativas do consumidor (FORNASIERO; ZANGIACOMI, 2013). Em número menor, tem-se o modelo focado na organização, padronização e sistematização das informações de projeto (MORETTI; BRAGHINI, 2017), como também na redução de tempo entre desenvolvimento do produto e venda (KINCADE; REGAN; GIBSON, 2007) e na exploração do conhecimento dos projetistas (MORRIS; ASHDOWN, 2018).

\begin{tabular}{|l}
\hline Modelo \\
\hline $\begin{array}{l}\text { Estrutura de desenvolvimento } \\
\text { de produtos para roupas de } \\
\text { desempenho }\end{array}$ \\
Criação de conhecimento de \\
projeto no processo de design \\
de vestuário de desempenho \\
Ferramenta metodológica para \\
contribuir no processo de \\
design de produtos de moda
\end{tabular}

Objetivo

Identificar as necessidades do consumidor, priorizando-as para alinhar-se às capacidades de fabricação, ou seja, integrar as necessidades do consumidor aos conceitos do produto.

Explorar os conhecimentos dos desenvolvedores de produtos de vestuário, quando se envolvem com os usuários.

Propor diretrizes projetuais que aprimoram e otimizam o processo de design de vestuário com foco em pessoas com mobilidade reduzida, proporcionando uma abordagem ergonômica na moda visando o conforto, segurança e
Quadro 4. Objetivos de aplicação dos modelos analisados

Fonte: Os autores. 
para pessoas com algum tipo de mobilidade reduzida

Modelo de referência para o processo de desenvolvimento de produtos de vestuário

Método orientado pelo usuário Modelo de crowdsourcing proposto para o desenvolvimento de novas ideias na indústria da moda

Mineração de regras de associação difusa

Metodologia de Implementação LPD para setor do vestuário

Modelo de referência CoReNet

Processo de desenvolvimento de produtos focado no consumidor

Modelo de Desenvolvimento de Produtos

Modelo de desenvolvimento proativo de produtos que integra os requisitos do consumidor Desenvolvimento de produto orientado ao usuário usabilidade, mas também considerando o bem-estar subjetivo individual, relacionado aos contextos culturais e sociais.

Apresentar um nível de detalhamento para sistematização, padronização e otimização do PDP do vestuário. Engloba desde o planejamento do projeto até o acompanhamento do produto no mercado, sugerindo gates necessários para acompanhar, controlar e otimizar o produto e o processo. Compreender e atender as necessidades dos usuários.

Gerar ideias inovadoras para empresas de moda a fim de corresponder melhor as necessidades dos seus clientes.

Descobrir a relação entre as características do produto e o comportamento de compra do cliente, com o objetivo de melhorar a eficácia e a eficiência do PDP de forma rápida. Criação de um repositório de conhecimento para a indústria do vestuário relacionando sistemas e práticas de desenvolvimento de produtos (alinhando-o à estratégia da empresa e levando em consideração o cliente, o meio ambiente e os aspectos de segurança e saúde).

Atender às necessidades e expectativas de grupos-alvo específicos - como idosos, obesos, deficientes ou diabéticos - para personalizar roupas e calçados funcionais e de alta qualidade, preço acessível e compatível com o meio ambiente.

Tornar o processo de desenvolvimento do produto do vestuário (mais concorrente e simultâneo) mais compacto, onde as atividades são realizadas no mesmo horário ou quase ao mesmo tempo. Objetivo redução do tempo entre desenvolvimento e a venda, ou seja, avançar as atividades tardias no processo de desenvolvimento de produto de vestuário.

Descrever as atividades funcionais (processos e práticas) que ocorrem no processo de desenvolvimento de produtos de varejo na Jamaica.

Trazer informações do consumidor para o desenvolvimento do produto.

O principal objetivo do método é satisfazer o interesse do usuário.

\section{DISCUSSÃO}

Foram identificados 13 modelos que auxiliam no PDP de vestuário, seja por meio de métodos, ferramentas ou quadros. Os modelos mais referenciados (ROSENBLAD-WALLIN, 1985; MAYPLUMLEE; LITTLE, 2006; KINCADE; REGAN; GIBSON, 2007; LEE et al., 2015; FORNASIERO; ZANGIACOMI, 2013), estão focados no processo inteiro do desenvolvimento, contemplando as fases de pré-desenvolvimento, desenvolvimento e pós-desenvolvimento, com exceção da 
proposta de May-Plumlee e Little (2006), que se dedica a fase de desenvolvimento e do Fornasiero e Zangiacomi (2013), que está focado no pré-desenvolvimento.

Os modelos identificados buscam auxiliar principalmente as fases de pré-desenvolvimento e desenvolvimento, de modo a proporcionar maior adequação das especificidades do usuário ao produto. Além disso, percebe-se a preocupação com a inserção do usuário em algumas etapas do processo, com o intuito de integrar suas necessidades aos requisitos de projetos, otimizando o processo de design, a fim de gerar ideias diferenciadas aos usuários. Este fato é corroborado pelas referências aos conceitos de Design Centrado no Usuário, Design Universal, Usabilidade, Ergonomia, Crowdsourcing e Engenharia do Produto. As empresas de vestuário estão enfrentando uma pressão considerável para reduzir seus custos e oferecer novos produtos e serviços, e, neste sentido a inovação é necessária para resolver esses problemas a fim de gerar novas ideias e novos avanços (TSE; CHAN, 2016).

Sanches (2008) aponta que o designer de moda precisa ter consciência sobre o perfil do usuário do produto em desenvolvimento, afinal é este universo que fornecerá as diretrizes para o direcionamento do projeto. Tse e Chan (2016) complementam que é necessário a compreensão das necessidades dos usuários para resolver os problemas referentes ao desenvolvimento de produtos de moda com uma abordagem sistemática de inovação. No entanto, devido à rápida tendência da moda, a indústria de vestuário precisa encurtar o tempo de ciclo de desenvolvimento de novos produtos, bem como atender às crescentes expectativas dos usuários (LEE, et al., 2015).

Em relação ao tempo de desenvolvimento, os modelos encontrados apresentam em média sete (7) fases, sendo que a menor quantidade de fases proposta é de 3 (MORETTI; BRAGHINI, 2017; JOHNSON-LESLIE; GASKILL, 2006) e a maior de 16 (MAY-PLUMLEE; LITTLE, 2006). As propostas abrangem principalmente as atividades de pesquisa (identificação dos requisitos do usuário), criação e detalhamento (geração das soluções), desenvolvimento (produção do vestuário), promoção e comercialização (apresentar a linha de produtos). Porém, é imprescindível levar em consideração as preferências dos usuários durante o processo de desenvolvimento dos produtos (LEE, et al., 2015). A este respeito a pesquisa identificou nove (9) dos modelos analisados que se preocupam com a incorporação do usuário em algumas fases do PDP.

No que se refere ao tipo de pesquisa, sete (7) documentos apresentaram o resultado de pesquisas empíricas, com apresentação de estudos de caso do modelo em uso. Dessa forma, como contribuições desses métodos pode-se destacar: coleta de informações do usuário (o que ele quer e precisa) para elencar em requisitos de projetos (TSE; CHAN, 2016); melhor satisfação do cliente, aumento da eficiência do PDP e maior desempenho para a empresa (LEE et al., 2015); gestão no PDP alinhado a estratégia da empresa, levando em consideração o cliente, o meio ambiente e aspectos de segurança e saúde (MALDONADO; RIVERA; CARO, 2014); engenharia simultânea como auxilio nas pressões do mundo competitivo e urgência de novidades para satisfazer o consumidor (KINCADE; REGAN; GIBSON, 2007); método (passos para o PDP de vestuário) aponta retorno eficaz sobre o investimento (JOHNSON-LESLIE; GASKILL, 2006); valorização das propriedades funcionais contribuindo para satisfazer o interesse do usuário pelo produto de vestuário (ROSENBLAD-WALLIN, 1985).

Quanto aos objetivos, os modelos buscam aprimorar o PDP priorizando a coleta de informações referente as reais necessidades dos usuários para a construção dos requisitos de projeto, a fim de gerar soluções adequadas as suas capacidades e limitações. Contudo, observou-se falta de propostas de modelos focados no desenvolvimento do produto de vestuário que considere o usuário em todo o processo de desenvolvimento, no sentido de guiar o projetista sobre como transformar os dados coletados em requisitos de projetos, considerando o conforto e a usabilidade para o desenvolvimento do produto de moda. Assim, apesar de muitos modelos 
abordarem a importância de se conhecer as reais necessidades do usuário, não é apresentada de forma detalhada e precisa como realizar este levantamento, tampouco como aplicar os resultados no desenvolvimento do projeto.

Além disso, observou-se falta de modelos ou ferramentas que auxiliem o projetista no PDP de vestuário considerando as propriedades ergonômicas e princípios de usabilidade aos produtos, principalmente na etapa de criação. Neste sentido, muitos modelos analisados, afirmam gerar parâmetros, diretrizes e princípios, para o desenvolvimento de produtos, porém não são apresentados de forma objetiva como podem auxiliar no PDP do vestuário, ficando muitas vezes com as reflexões geradas nos estudos de casos realizados. Isso pode estar relacionado ao fato de que a indústria de confecção do vestuário está sujeita à influência da moda, conferindo ao processo de PDP do vestuário grande dinamismo e velocidade no tempo de desenvolvimento (MORETTI; BRAGHINI, 2017).

\section{CONCLUSÃO}

O Processo de Desenvolvimento de Produtos é estratégico para as empresas de confecção do vestuário, afinal, tem por objetivo transformar dados e possibilidades técnicas em oportunidades de mercado, visando auxiliar em projetos de produtos. Assim, a escolha de métodos e ferramentas a serem empregadas no setor de desenvolvimento é de suma importância, visto que podem determinar características ao produto final, a citar: design, qualidade, desempenho, custo, tempo de produção, aceitação no mercado, conforto, usabilidade, entre outros. Mediante essas características, é fundamental que o setor da moda inove além do design no produto, como nos processos gerenciais e operacionais, buscando maneiras de serem flexíveis, eficientes e mais direcionados em suas previsões e produção de produtos.

Como contribuição este artigo se propôs a identificar e apresentar os modelos existentes sobre o PDP de vestuário, a fim de disseminar os estudos sobre a temática, para beneficiar pesquisadores, professores, e discentes dos cursos de Design de Moda em seus projetos. Além disso, permitiu fornecer um panorama geral da contribuição desses modelos e como podem ser utilizados por profissionais das empresas de confecção, que poderão melhorar a organização dos seus processos de desenvolvimento de produtos de vestuário, bem como contribuir com o próprio consumidor que terá a sua disposição peças de vestuário que se adequam melhor as suas necessidades e proporcionam maior satisfação, qualidade e confiabilidade.

Quanto as contribuições teóricas deste artigo, identificou-se novas lacunas de pesquisa científicas, a citar: novos modelos de desenvolvimento do produto de vestuário que considere o usuário em todo o processo de desenvolvimento; utilização de propriedades ergonômicas e princípios de usabilidade para o desenvolvimento do produto de vestuário, principalmente na etapa de criação; dificuldade de identificação de demandas e compreensão do público-alvo; geração de planos de trabalho e gestão visual de projetos, que auxiliem as etapas de desenvolvimento do produto (criação e modelagem), abordando as questões técnicas, ergonômicas e estéticas. E por fim, na etapa de pós-desenvolvimento (avaliação do produto com ou sem o usuário) também é uma oportunidade para estudos.

Como futuros estudos, pretende-se realizar um levantamento semelhante em bancos de teses e dissertações, nacionais e internacionais, bem como a realização de levantamentos in loco, mediante entrevistas com profissionais da área de moda, pesquisadores e professores, bem como alunos de graduação. Com esses levantamentos será possível gerar novas ferramentas que venham a contribuir com o PDP, e atender às necessidades criadas pelo uso pretendido. 


\section{Agradecimentos}

Agradecemos ao Programa de Pós-graduação em Design da UFSC (PósDesign/UFSC), ao Programa de Pós-Graduação em Engenharia de Produção (PPGEP/UFSC), ao Núcleo de Gestão de Design e Laboratório de Design e Usabilidade (NGD-LDU/UFSC), à Coordenação de Aperfeiçoamento de Pessoal de Nível Superior- Brasil (CAPES) - Código de Financiamento 001, e ao Conselho Nacional de Desenvolvimento Científico e Tecnológico (CNPq).

\section{Referências Bibliográficas}

AYAĞ, Zeki. A fuzzy AHP-based simulation approach to concept evaluation in a NPD environment. IIE transactions, v. 37, n. 9, p. 827-842, 2005.

BACK, Nelson et al. Projeto integrado de produtos: planejamento, concepção e modelagem. Barueri - São Paulo: Manole, 2008.

FORNASIERO, Rosanna; ZANGIACOMI, Andrea. A structured approach for customised production in SME collaborative networks. International Journal of Production Research, v. 51, n. 7, p. 2110-2122, 2013.

GUNESOGLU, Sinem; MERIC, Binnaz. The analysis of personal and delay allowances using work sampling technique in the sewing room of a clothing manufacturer. International Journal of Clothing Science and Technology, v. 19, n. 2, p. 145-150, 2007.

JOHNSON-LESLIE, Natalie; GASKILL, LuAnn R. Small business apparel retailing in Jamaica: an exploratory investigation into product development processes and practices. The Qualitative Report, v. 11, n. 3, p. 417-449, 2006.

KINCADE, Doris H.; REGAN, Cynthia; GIBSON, Fay Y. Concurrent engineering for product development in mass customization for the apparel industry. International Journal of Operations \& Production Management, v. 27, n. 6, p. 627-649, 2007.

LEE, Carmen Kar Hang et al. Fuzzy association rule mining for fashion product development. Industrial Management \& Data Systems, v. 115, n. 2, p. 383-399, 2015.

MALDONADO, Diana Isabel Barón; RIVERA, Leonardo; CARO, Mauricio. Improvement of new product development process in apparel SMEs using lean and life cycle. In: Proceedings of the XIX Summer School Francesco Turco, Issue Industrial Mechanical Plants - SSD INGIND17, Ancona, p.379-384. 2014.

MAY-PLUMLEE, Traci.; LITTLE, Trevor J. No-interval coherently phased product development model for apparel. International Journal of Clothing Science and Technology, v. 10 n. 5, p. 342-364, 1998.

MAY-PLUMLEE, Traci; LITTLE, Trevor J. Proactive product development integrating consumer requirements. International Journal of Clothing Science and Technology, v. 18, n. 1, p. 53-66, 2006.

MEHTÄLÄ, Joanna et al. A crowdsourcing model for new idea development in the fashion industry. In: 2016 IEEE International Conference on Service Operations and Logistics, and Informatics (SOLI). IEEE, 2016. p. 29-36.

MORETTI, Isabel Cristina; BRAGHINI, Aldo Junior. Reference model for apparel product development. Independent Journal of Management \& Production, v. 8, n. 1, p. 232-262, 2017.

MORRIS, Kristen D.; ASHDOWN, Susan P. Partnerships in Practice: Producing New Design Knowledge with Users When Developing Performance Apparel Products. Fashion Practice, v. 10, n. 3, p. 328353, 2018. 
Thiago Varnier

thiagovarnier1@gmail.com

Diego de Castro Fettermann dcfettermann@gmail.com

Giselle S. A. D. Merino

gisellemerino@gmail.com
Processo de desenvolvimento de produtos no vestuário: uma revisão sistemática de modelos de auxílio à prática projetual de produtos de moda

NAKAYAMA, Gabriela Y.; MARTINS, Laura B. Fashion Design Methodology Tools in Products' Development for People with Disabilities and Low Mobility. In: BAGNARA S., TARTAGLIA R., ALBOLINO S., ALEXANDER T., FUJITA Y. (eds) Proceedings of the 20th Congress of the International Ergonomics Association. IEA 2018. Advances in Intelligent Systems and Computing, vol 824. Springer, Cham, p. 1699-1704, 2018.

PICHLER, Rosimeri Franck; MERINO, Giselle Schmidt Alves Díaz. Design e Tecnologia Assistiva: uma revisão sistemática de modelos de auxílio à prática projetual de dispositivos assistivos. Estudos em Design, v. 25, n. 2, 2017.

ROSENBLAD-WALLIN, Elsa. User-oriented product development applied to functional clothing design. Applied ergonomics, v. 16, n. 4, p. 279-287, 1985.

SAMPAIO, Rosana F.; MANCINI, Marisa C. Systematic review studies: a guide for careful synthesis of the scientific evidence. Brazilian Journal of Physical Therapy, v. 11, n. 1, p. 83-89, 2007.

SILVA, RK Jayamali De; RUPASINGHE, Thashika D. A new apparel product development framework for performance clothing industry. International Journal of Product Development, v. 22, n. 4, p. 276 292, 2018.

TREPTOW, Doris. Inventando moda: planejamento de coleção. Doris Treptow -5 ed. São Paulo: Edição da autora, 2013.

TSE, Florence, TF; CHAN, Catherine YP. New approach for fashion design: Case study of employing user-oriented method to design mother-to-be party dress. Research Journal of Textile and Apparel, v. 20, n. 1, p. 53-60, 2016. 